\title{
Statistics of defect motion in spatiotemporal chaos in inclined layer convection
}

\author{
Karen E. Daniels ${ }^{\text {a) }}$ and Eberhard Bodenschatz ${ }^{\text {b) }}$ \\ Laboratory of Atomic and Solid State Physics, Cornell University, Ithaca, New York 14853
}

(Received 4 November 2002; accepted 20 November 2002; published 17 January 2003)

\begin{abstract}
We report experiments on defect-tracking in the state of undulation chaos observed in thermal convection of an inclined fluid layer. We characterize the ensemble of defect trajectories according to their velocities, relative positions, diffusion, and gain and loss rates. In particular, the defects exhibit incidents of rapid transverse motion which result in power law distributions for a number of quantitative measures. We examine connections between this behavior and Lévy flights and anomalous diffusion. In addition, we describe time-reversal and system size invariance for defect creation and annihilation rates. (C) 2003 American Institute of Physics. [DOI: 10.1063/1.1536330]
\end{abstract}

Topological defects within patterns are observed in many systems to move in a spatiotemporally chaotic fashion. We examine the motion of such defects within a defectturbulent state observed in thermal convection of an inclined fluid layer. We characterize the trajectories of the defects both by analogy to fluid turbulence-velocity distributions, diffusion, and power spectra-and by properties dependent upon the topological characteristics of the defects: pair creation/annihilation and interactions.

\section{INTRODUCTION}

Nonequilibrium systems with similar symmetries often form patterns which appear to be universal in spite of having been formed by different physical mechanisms. ${ }^{1}$ In particular, reduced descriptions of the patterns often quantify the similarities in behavior so that understanding of one system can lead to insights in multiple systems. A class of spatiotemporally chaotic states exhibiting defect-mediated turbulence ${ }^{2}$ has been found in such diverse systems as wind-driven sand, electroconvection in liquid crystals, ${ }^{3}$ nonlinear optics, ${ }^{4}$ fluid convection, ${ }^{5,6}$ and autocatalytic chemical reactions. ${ }^{7}$ In many cases, such systems have been modeled via the complex Ginzburg-Landau equation. ${ }^{8-12}$ These various defect turbulent patterns are characterized by an underlying striped state which contains dislocations (point defects) where the stripes dead-end within the pattern. Locally, the defects distort the orientation and wavenumber of the stripes and the nucleation, motion, and annihilation of the defects constitute a spatiotemporally chaotic system. An example from inclined layer convection is shown in Fig. 1.

Previous work on defect turbulence has focused both on snapshots of such patterns $s^{3,4,10}$ and the dynamics and interaction. ${ }^{8,9,11}$ However, there are numerous open questions about defect turbulence: characterization of the defect motions, interactions between the defects, and the extent to

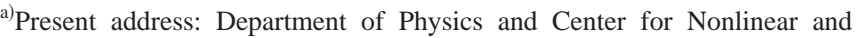
Complex Systems, Duke University, Durham, North Carolina 27708; electronic mail: ked@phy.duke.edu

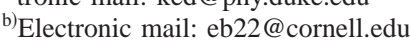

which the analogy to turbulence is appropriate. The degree to which such characteristics are similar in different defectturbulent systems remains to be explored.

Investigations of pattern formation in variants of Rayleigh-Bénard convection (RBC) have been particularly fruitful. ${ }^{13}$ The state of undulation chaos (shown in Fig. 1 and described in Refs. 14-16) observed in inclined layer convection (Fig. 2) exhibits defect turbulence and is well suited to investigations on the dynamics of defects since spatially extended systems and fast time scales are experimentally accessible. This allows for tracking of point defects through their creation, motion, and annihilation. In the observed pattern, the stripes contain undulations as well as defects; both are spatiotemporally chaotic (further characterization to be published in Ref. 16).

A number of features stand out in the defect trajectories we observe in undulation chaos. As exemplified in Fig. 3, the inherent anisotropy (due to the inclination) is apparent, with the trajectory meandering preferentially in the transverse direction. Occasionally, there is a rapid burst of transverse motion as marked by the black diamonds, corresponding to a tearing of the pattern across the rolls. Such behavior appears be related to Lévy flights ${ }^{17-19}$ for which distributions of step sizes display power laws. Furthermore, we are able to study the ensemble of trajectories to gain insight into defect pair creation, interaction, and annihilation.

\section{INCLINED LAYER CONVECTION}

In inclined layer convection (ILC), a thin fluid layer heated from one side and cooled from the other is tilted by an angle $\gamma$; the system is anisotropic due to the resulting shear flow (see Fig. 2). The fluid becomes unstable above a critical temperature difference $\Delta T_{c}$. At fixed $\gamma$, we describe how far the system is above the onset of convection via the nondimensional driving parameter $\epsilon \equiv\left[\Delta T / \Delta T_{c}(\gamma)\right]-1$.

At low angles of inclination, buoyancy provides the primary instability (analogous to RBC) and the convection rolls are aligned with the shear flow (longitudinal) both at onset and for the secondary transition to undulation chaos. ${ }^{20}$ Over a range of intermediate angles $\left(15^{\circ} \leqslant \gamma \leqslant 70^{\circ}\right.$ for Prandtl 


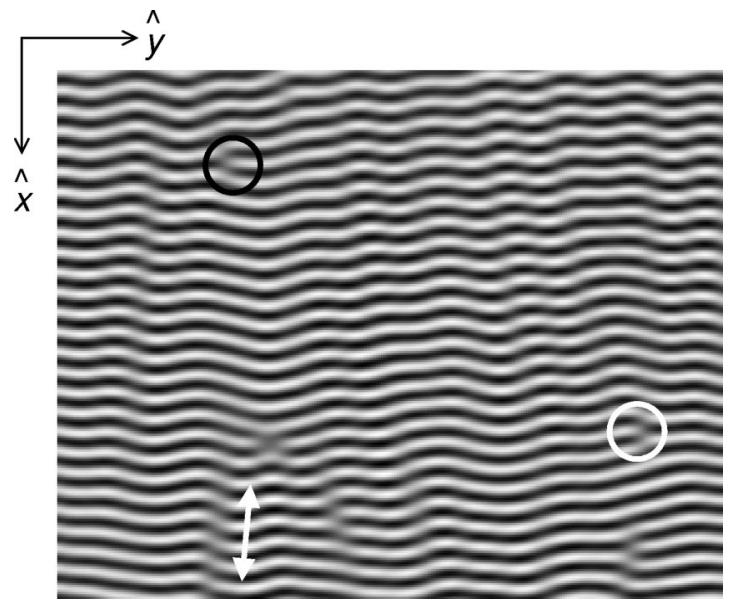

FIG. 1. Sample Fourier-filtered shadowgraph image of inclined layer convection at $\epsilon=0.08$ and $\gamma=30^{\circ}$. Black circle encloses a positive defect; white, a negative. Arrow indicates tearing region of low-amplitude convection. Uphill direction is at left side of page. Region shown is the subregion of size $51 d \times 63 d$ used for analysis.

number $\sigma \approx 1$ ), the longitudinal rolls become unstable to undulation chaos above $\epsilon \approx 0.01$. It is this defect turbulent state which we investigate; some of its properties have been previously described in other work. ${ }^{15,16}$ Above $\epsilon \approx 0.1$, there is a further transition to a state of competing ordered undulations and undulation chaos. We examine trajectories from both sides of this transition.

The apparatus used in this experiment is of the type described in de Bruyn et al., ${ }^{21}$ modified to allow for inclination. The fluid used was $\mathrm{CO}_{2}$ at a pressure of $(56.5 \pm 0.01)$ bar regulated to \pm 0.005 bar with a mean temperature of $(28 \pm 0.05)^{\circ} \mathrm{C}$ regulated to $\pm 0.0003{ }^{\circ} \mathrm{C}$. As determined from a materials properties program, ${ }^{21}$ the Prandtl number was $\sigma=1.140 \pm 0.001$. A cell of height $d=(388 \pm 2) \mu \mathrm{m}$ and dimensions $203 d \times 100 d$ was used, for which the vertical diffusion time was $\tau_{v}=d^{2} / \kappa=(1.532 \pm 0.015) \mathrm{s}$. The fluid was weakly non-Boussinesq conditions: $Q=0.8$, as described in Ref. 13 for horizontal fluid layers. All experiments were performed at a fixed inclination of $\gamma=30^{\circ}$, within the regime of buoyancy-instability. Images of the convection pattern were collected using a digital CCD camera, via the usual shadowgraph technique. ${ }^{21,22}$ Images were collected at 3 frames per second in one of two formats. Six-hour $\left(2 \times 10^{4} \tau_{v}, 80000\right.$ frames) continuous runs of data were obtained at two values of $\epsilon$ : 0.08 (four runs) and 0.17 (two runs). For 17 values of $\epsilon$ between 0.04 and 0.22 , short runs with 100 images were collected, separated by at least $100 \tau_{v}$ for statistical indepen-
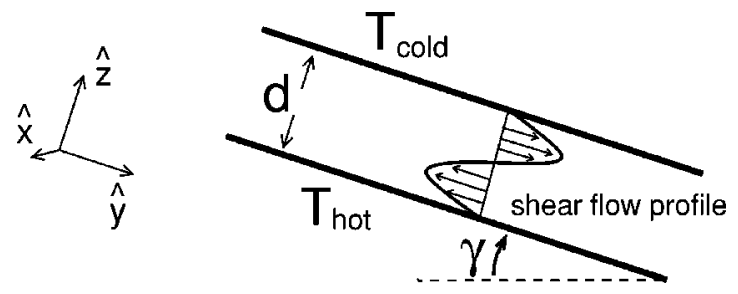

FIG. 2. Schematic of inclined layer convection with associated coordinate system. $\Delta T \equiv T_{\text {hot }}-T_{\text {cold }}$.
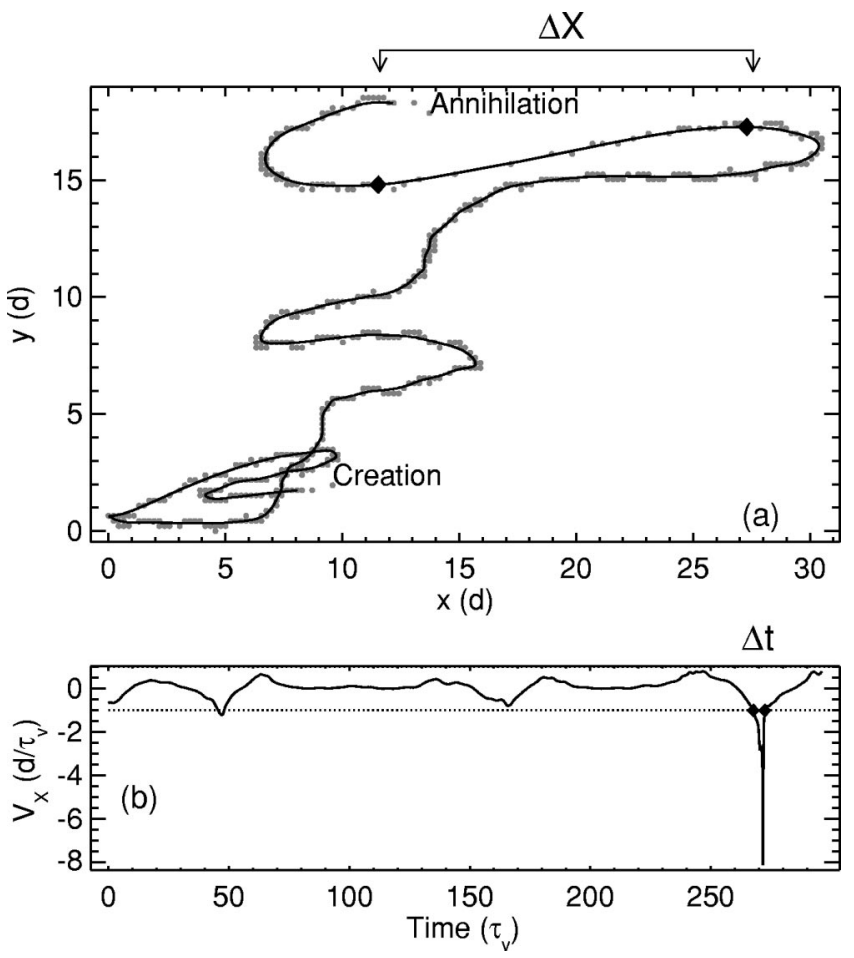

FIG. 3. (a) Example of a long trajectory for a defect with positive topological charge at $\epsilon=0.08$. Positive $\hat{\mathbf{y}}$ indicates a downslope direction (see Fig. 1). (b) Corresponding transverse velocity. The symbols $\downarrow$ mark the ends of a flight with displacement $\Delta X$ and duration $\Delta t$. Dotted line is the trigger velocity for detecting flights.

dence. At each of these $\epsilon$, at least 400 repeats (up to 600 for the lowest values of $\epsilon$ ) were performed. Each value of $\epsilon$ was reached by a quasistatic temperature increase from below. In addition, a run with quasistatic temperature decreases was performed between $\epsilon=0.12$ and $\epsilon=0.06$ to check for hysteresis, which was not observed. Only data from the central homogeneous region of dimension $51 d \times 63 d$ were utilized during the analysis unless noted otherwise; see Ref. 16 for details on the choice of this region. Size-dependent effects of this region are discussed here.

\section{DEFECT TRAJECTORIES}

Here we consider only the defects themselves - an ensemble of moving, interacting, charged "particles" — and not the underlying pattern. By reducing the data to a set of defect trajectories of known charge, birth, and death we can observe how these particles move individually and as an ensemble. Single defects may enter or leave through the edges of the system, or be created/annihilated in pairwise events between defects of opposite topological charge. ${ }^{15}$ During their lifetimes, the defects have positions and velocities which may be related both to the underlying pattern and to the presence of other defects. We examine properties averaged over these effects, and also isolate some effects due to the latter.

The topological defects are located at points where a roll pair ends within the pattern (see Fig. 1). At these points, there is a phase discontinuity and the topological charge is determined from the phase jump along a contour around the defect, 


$$
\oint \nabla \phi \cdot d \mathbf{s}=n 2 \pi,
$$

The phase field $\phi(x, y)$ is determined from Fourierdemodulated images for which the complex field $\psi(x, y)$ $=|A(x, y)| e^{i \phi(x, y)}$ has been reconstructed. We detect the locations of defects by finding points where $\mathfrak{R}(\psi)=0$ and $\Im(\psi)=0$ and a phase discontinuity occurs. (Details of this technique are described in Ref. 16.) In undulation chaos, we observe only defects with $n= \pm 1$; examples of each topological charge are marked by circles in Fig. 1.

After the defects were detected in each image, the defect locations were connected to form trajectories. This was done by matching each defect already on a trajectory to its closest same-signed neighbor in the subsequent frame. If the reverse process also agreed on the match, then the subsequent defect was added to the list of defects located on that trajectory. Similarly, the ends of positive and negative defect trajectories were matched up to obtain the locations of all pair creations and annihilations. When the analysis was completed, broken trajectories (those missing a creation or annihilation) were rejoined.

Since the defect positions were determined only to the nearest pixel, the data were later smoothed to interpolate the coordinates along the trajectory. Each $x_{i} \equiv x\left(t_{i}\right)$ and $y_{i} \equiv y\left(t_{i}\right)$ along a trajectory was replaced with the weighted average of its neighbors, using the Gaussian weighting function

$$
\begin{aligned}
w_{i j} \equiv & \frac{1}{\sigma_{x} \sigma_{y} \sigma_{t}(2 \pi)^{3 / 2}} \exp \left[-\frac{\left(x_{i}-x_{j}\right)^{2}}{2 \sigma_{x}^{2}}\right] \\
& \cdot \exp \left[-\frac{\left(y_{i}-y_{j}\right)^{2}}{2 \sigma_{y}^{2}}\right] \cdot \exp \left[-\frac{\left(t_{i}-t_{j}\right)^{2}}{2 \sigma_{t}^{2}}\right] .
\end{aligned}
$$

This resulted in a smoothed trajectory with coordinates $\tilde{x}_{i}$ $=\sum w_{i j} x_{j}$ and $\tilde{y}_{i}=\sum w_{i j} y_{j}$ (tildes will be dropped hereafter for convenience). The appropriate widths $\sigma$ in $w_{i j}$ were determined by examining a range of parameters and finding convergence for $\sigma_{x}=\sigma_{y}=2 d$ and $\sigma_{t}=2 \tau_{v}$. The advantage of this weighting method is that it automatically adjusts the fit length so that along faster-moving segments of the trajectory we average fewer data points than along slower-moving segments.

From the trajectories $x(t)$ and $y(t)$, we applied a local weighted linear fit (again using $w_{i j}$ ) at each point along the trajectory to obtain the corresponding velocities $v_{x}(t)$ and $v_{y}(t)$. We also determined $v \equiv \sqrt{v_{x}^{2}+v_{y}^{2}}$ and $\theta \equiv \arctan \left(v_{x} / v_{y}\right)$, corresponding to a downslope $(\hat{\mathbf{y}})$ direction at $\theta=0^{\circ}$. A raw trajectory is shown in Fig. 3, along with the smoothed trajectory and the transverse velocity component $v_{x}(t)$.

Defect turbulence in inclined layer convection is anisotropic, with $v_{x}$ corresponding to glide motion across the rolls and $v_{y}$ to climb motion along the rolls. These are fundamentally different behaviors; the former adjusts the orientation of the rolls, and the later the wavenumber. As expected, the decomposed motions are found to be poorly correlated with each other, with a linear correlation coefficient $R^{2}=0.16$ for
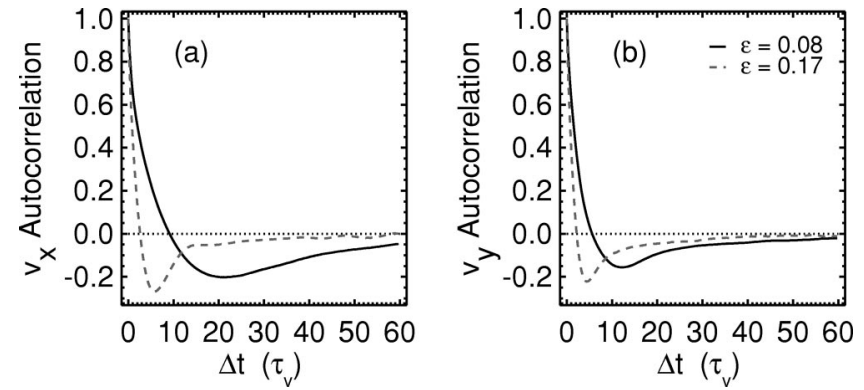

FIG. 4. Velocity autocorrelation in (a) transverse and (b) longitudinal directions at $\epsilon=0.08$ and $\epsilon=0.17$. Data are shown for positive defects; results for negative defect trajectories were similar.

positive defect trajectories and $R^{2}=0.12$ for negative. Therefore, we separate all defect positions and motions into their two components and examine each direction independently.

Defect velocities are observed to be correlated over time scales of less than $\approx 10 \tau_{v}$, as determined from temporal autocorrelation functions of $v_{x}(t)$ and $v_{y}(t)$. The autocorrelations are plotted in Fig. 4, averaged over the ensemble of trajectories. The defect motions exhibit short-range order, with exponential decay during the time before the zerocrossing.

\section{DEFECT MOTION}

We characterize the meanderings of the defect via its central moments, represented by integer powers of the deviations from its mean value. For trajectories $x_{i}(t)$ with mean $\overline{x_{i}(t)}$, the $n$th moment is given by the quantity

$$
\mu_{n}(t) \equiv\left\langle\left(x_{i}(t)-\overline{x_{i}}\right)^{n}\right\rangle,
$$

where $\langle\cdot\rangle$ represents an ensemble average, and a similar calculation can be made for the $y(t)$. For normal diffusion or random walks, $\mu_{n} \propto t^{n / 2}$, where $\mu_{2}=2 D t$ provides a value $D$ for the diffusion constant.

Investigations in a broad variety of situations (see, for example, Refs. 17-19,23, and references therein) have found anomalous diffusion instead, where $\mu_{2}=2 D t^{\alpha}$. For $\alpha<1$ a system is said to be subdiffusive, $\alpha>1$ is superdiffusive, and $\alpha=2$ is ballistic transport. Anomalous diffusion is frequently associated with Lévy walks/flights, in which the PDFs of the lengths and durations of the flights are power laws and have infinite moments. Since the central limit theorem no longer holds in such cases, the probability of long jumps (waits) will enhance (retard) the diffusive behavior. Similarly, the power spectrum $S(\omega)$ of the associated velocities will exhibit power law behavior as well. Some recent examples of such behavior in fluid dynamics include tokamak density fluctuations ${ }^{24}$ and particle motion in two-dimensional rotating fluid flows. ${ }^{25}$ Defect random walks have also been considered for models of intracellular $\mathrm{Ca}^{2+}$ dynamics, ${ }^{8}$ in which subdiffusion was observed.

In Figs. 5 and 6 we calculate moments for those values of $t$ at which 100 or more trajectories have at least that duration. We look for behavior of the type $\mu_{2} \propto t^{\alpha}, \mu_{4} \propto t^{\beta}$, and $\mu_{6} \propto t^{\gamma}$ in both the $\hat{\mathbf{x}}$ and $\hat{\mathbf{y}}$ directions. In the variance $\left(\mu_{2}\right)$, superdiffusive behavior is evident at both $\epsilon$, particularly in $\hat{\mathbf{x}}$ 

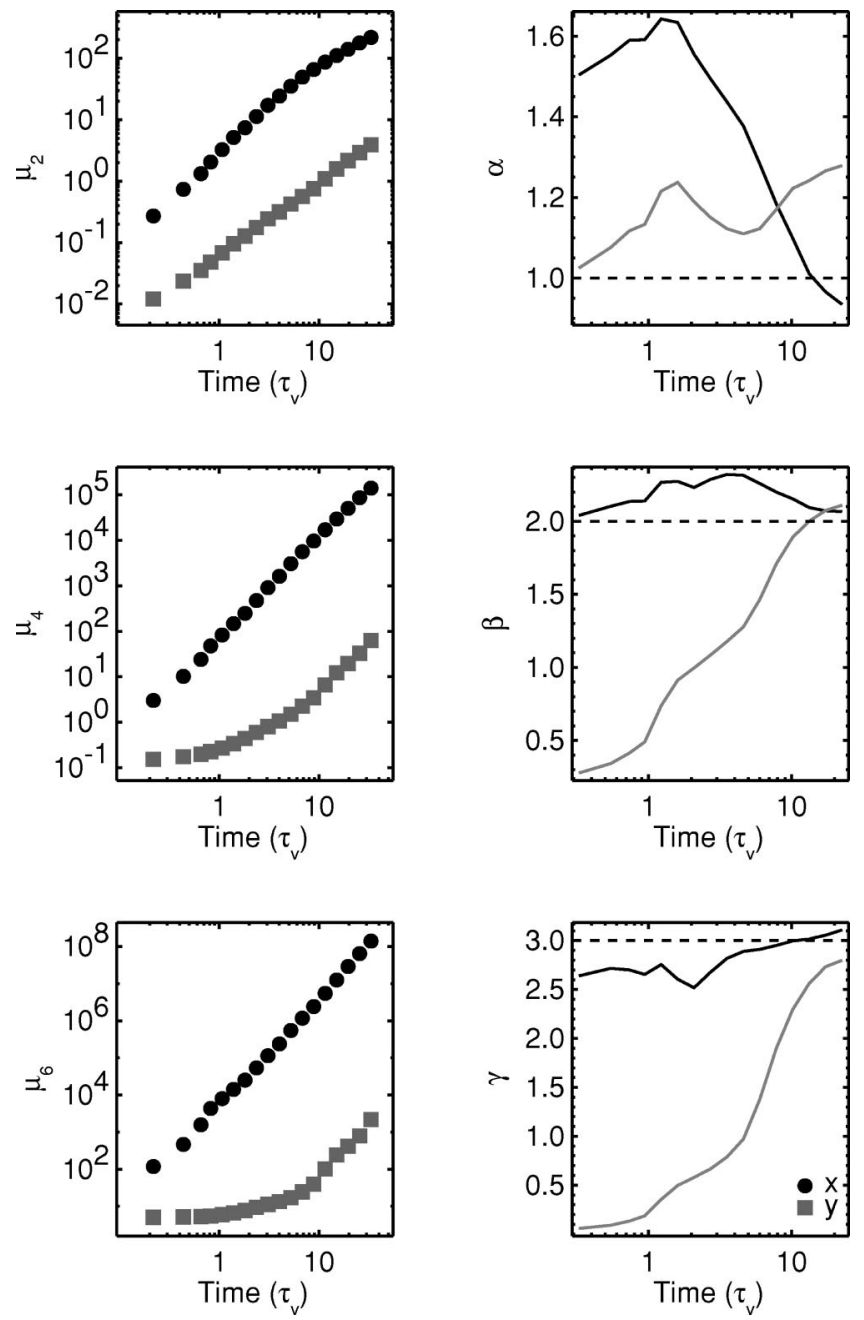

FIG. 5. Displacement moments [Eq. (3)] and local fits to the associated exponents in $\hat{\mathbf{x}}$ (black) and $\hat{\mathbf{y}}$ (gray) directions at $\epsilon=0.08$. Dashed lines are values for normal diffusion or random walks, $\mu_{n} \propto t^{n / 2}$. Data are shown for positive defects; results for negative defects were similar.

where $\alpha \approx 1.5$. The exponents for $\hat{\mathbf{y}}$ are lower but also indicate superdiffusion. The exponents of $\mu_{4}$ and $\mu_{6}$ do not suggest superdiffusion in $\hat{\mathbf{x}}$, and the $\hat{\mathbf{y}}$ behavior does not follow a power law.

We locate defects flights as the portions of the trajectories which occur while $\left|v_{x}\right|>1 d / \tau_{v}$. An example flight is shown in Fig. 3. For each of these flights, we determine the duration $\Delta t$ and displacement $\Delta X$. Distributions of these values are shown in Fig. 7. Due to the constraints of a finite system size, the maximum observable $\Delta X$ is limited. Nonetheless, the distribution of flight times $\Delta t$ is observed to have behavior consistent with a power law tail of exponent approximately -3 . For $\Delta X$, behavior suggestive of a power law with exponent approximately -4 is observed, indicating that there is not a characteristic velocity associated with the flights.

Physically, these flight events correspond to a tearing of the rolls when the pattern shifts along a line in the $\hat{\mathbf{x}}$ direction, so that each roll broken in the process rejoins with the one next to it. Such a tearing line is visible next to the white arrow in Fig. 1, where there is weaker convection along the
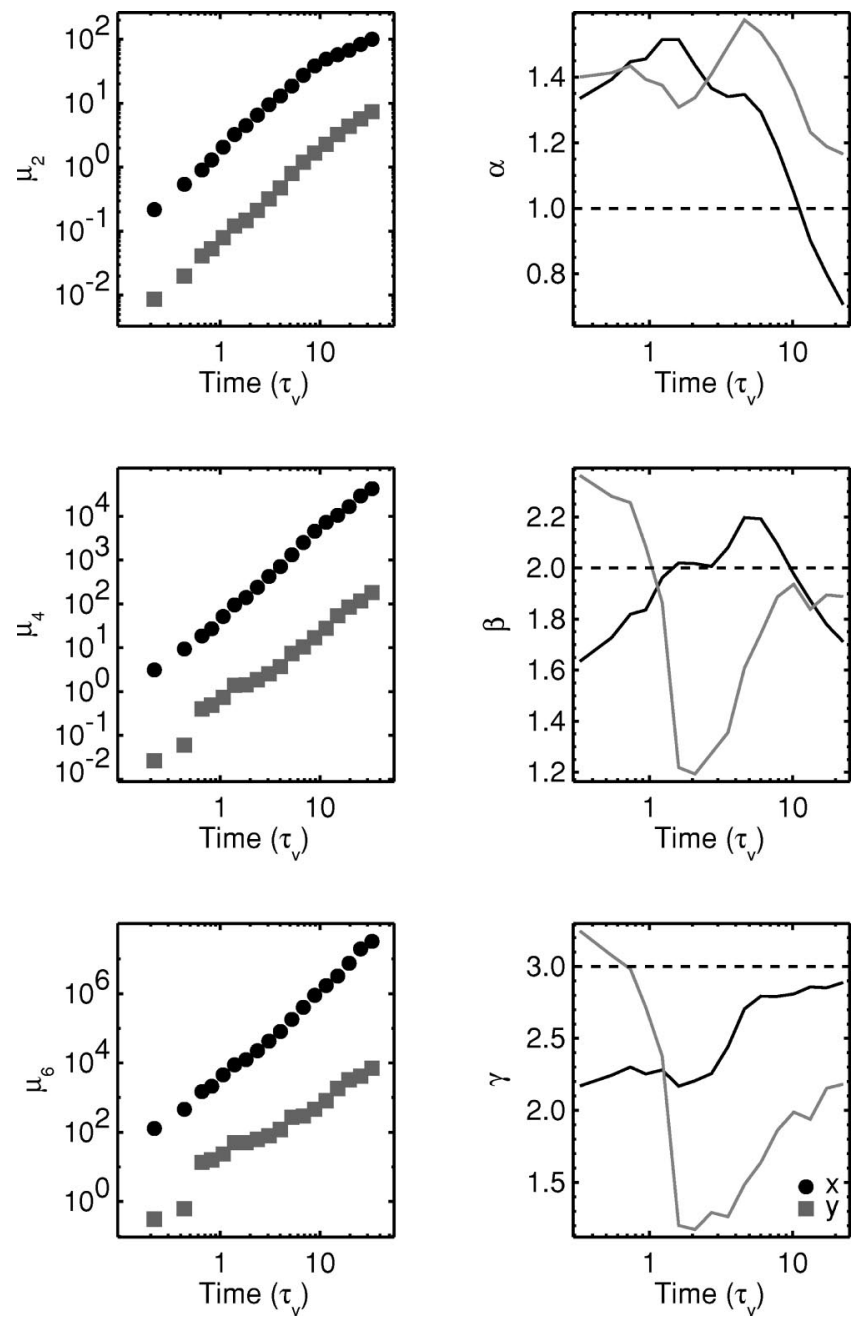

FIG. 6. Displacement moments [Eq. (3)] and local fits to the associated exponents in $\hat{\mathbf{x}}$ (black) and $\hat{\mathbf{y}}$ (gray) directions at $\epsilon=0.17$. Dashed lines are values for normal diffusion or random walks, $\mu_{n} \propto t^{n / 2}$. Data are shown for positive defects; results for negative defects were similar.

slopes of the undulations. Since each roll must move by only $2 d$ to reconnect with the next roll, defects can be transported rapidly from one end of the tear line to the other.

By analogy to fluid turbulence, we plot probability distribution functions (PDFs) and power spectra of the defect velocities. Figures 8 and 9 show the results for distributions in $\hat{\mathbf{x}}$ and $\hat{\mathbf{y}}$. The PDFs of $v_{x}$ are independent of topological charge and show steep power law tails with an exponent of approximately -3.5 . This is consistent with the results obtained for defect flights displacements and durations. As can be seen in Figs. 8 and 9, the peak of the distributions have a Gaussian shape, indicative of smoothing due to noise. Each distribution is also of a shape described by Tsallis statistics of the type described in Ref. 26. The PDFs for $v_{y}$ show lower characteristic velocities and (particularly at $\epsilon=0.08$ ) dependence on the topological charge. Figure 10 shows a complementary picture in $(v, \theta)$ space via a two-dimensional histogram. The $\hat{\mathbf{x}}$ flights are now visible as the ridges at approximately $\theta= \pm 90^{\circ}$. This transverse direction not only comprises most of the defect motion, but is also the direction along which motion is the fastest. 


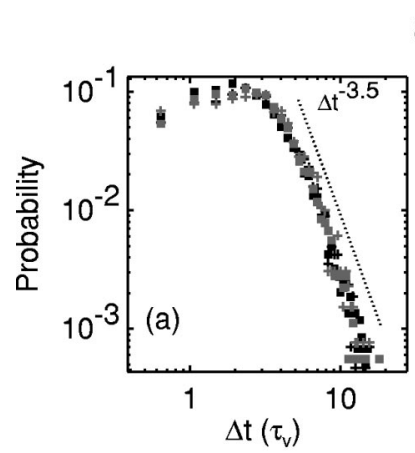

$\varepsilon=0.08$

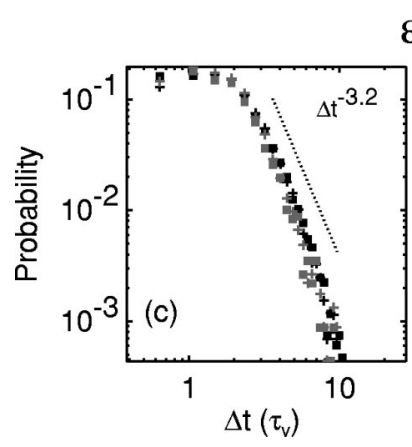

$\varepsilon=0.17$
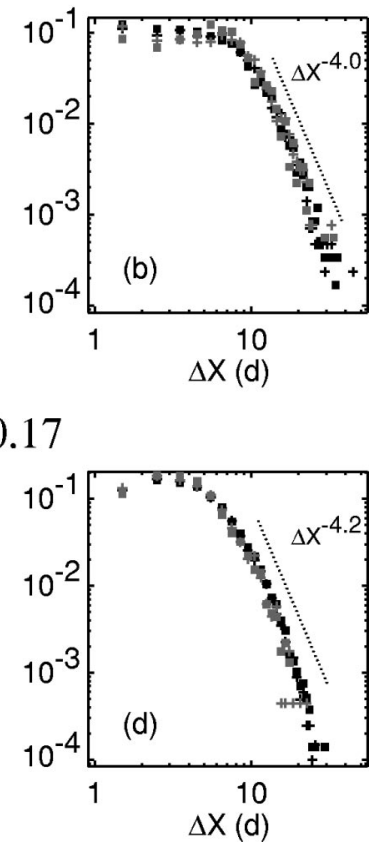

FIG. 7. Distributions of flight (a,c) durations $\Delta t$ and (b,d) displacements $\Delta X$ at $\epsilon=0.08$ and $\epsilon=0.17$. Positive defects are + , negative defects are squares, black points are from entire trajectories, and gray from segments within the homogeneous subregion. Dotted lines represent fit region for power laws.

A second effect visible in Figs. 8, 9, and 10 is an asymmetry in the $\pm \hat{\mathbf{y}}$ behavior which shifts the $(v, \theta)$ peaks in the upslope or downslope direction, away from $\pm 90^{\circ}$. Although the $\epsilon=0.17$ defects are more isotropic in their motion than
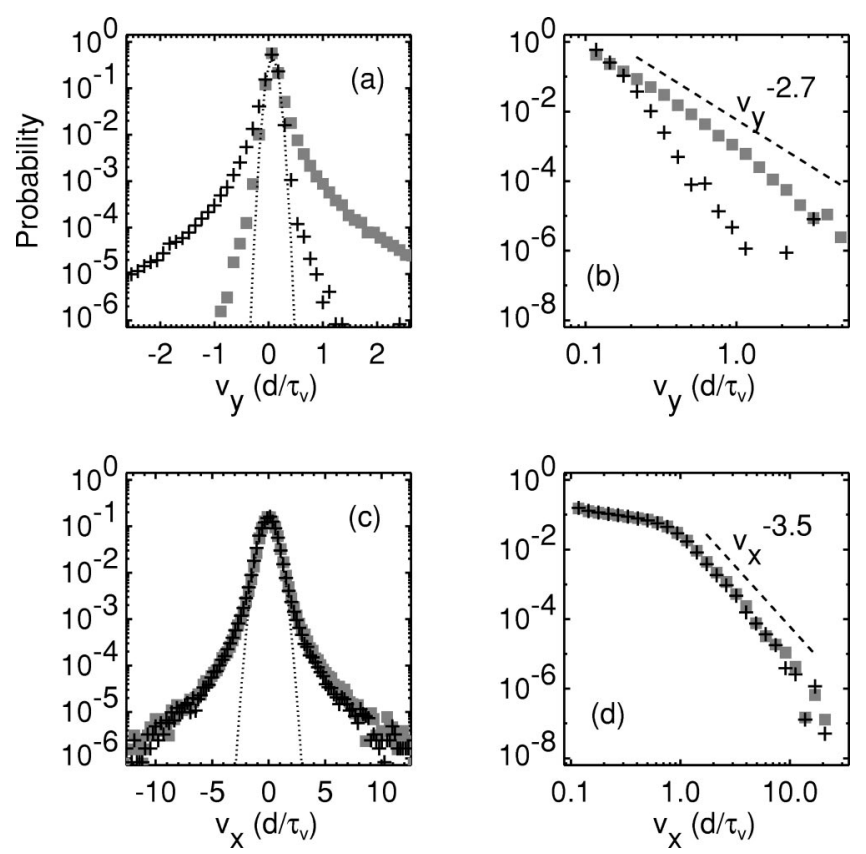

FIG. 8. (a,b) Longitudinal and (c,d) transverse velocity PDFs at $\epsilon=0.08$ on $\log -\log$ and semilog (for $v>0$ ) axes. Positive defects are shown with black + and negative defects with gray squares. Approximately $10^{6}$ defect velocity measurements were used to determine the probabilities in each graph. Dotted lines are Gaussian fits to whole data, with standard deviations $\sigma_{y}$ $=0.08$ and $\sigma_{x}=0.59$. Dashed lines represent fit region for power laws.
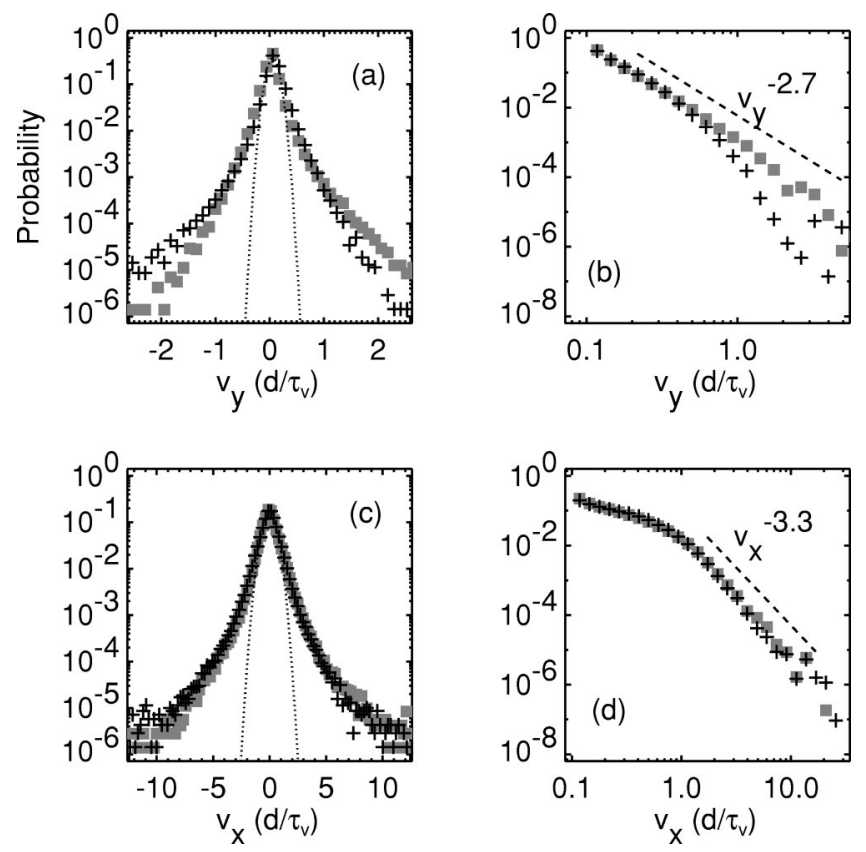

FIG. 9. (a,b) Longitudinal and (c,d) transverse velocity PDFs at $\epsilon=0.17$ on $\log -\log$ and semilog (for $v>0$ ) axes. Positive defects are shown with black + and negative defects with gray squares. Approximately $10^{6}$ defect velocity measurements were used to determine the probabilities in each graph. Dotted lines are Gaussian fits to whole data, with standard deviations $\sigma_{y}$ $=0.10$ and $\sigma_{x}=0.51$. Dashed lines represent fit region for power laws.

the $\epsilon=0.08$ defects, in both cases the negative defects prefer the downslope direction over the upslope, and the positive defects prefer the upslope. In fact, Figs. 8 and 9 show that this asymmetry is the dominant behavior at lower $\epsilon$; it is rare

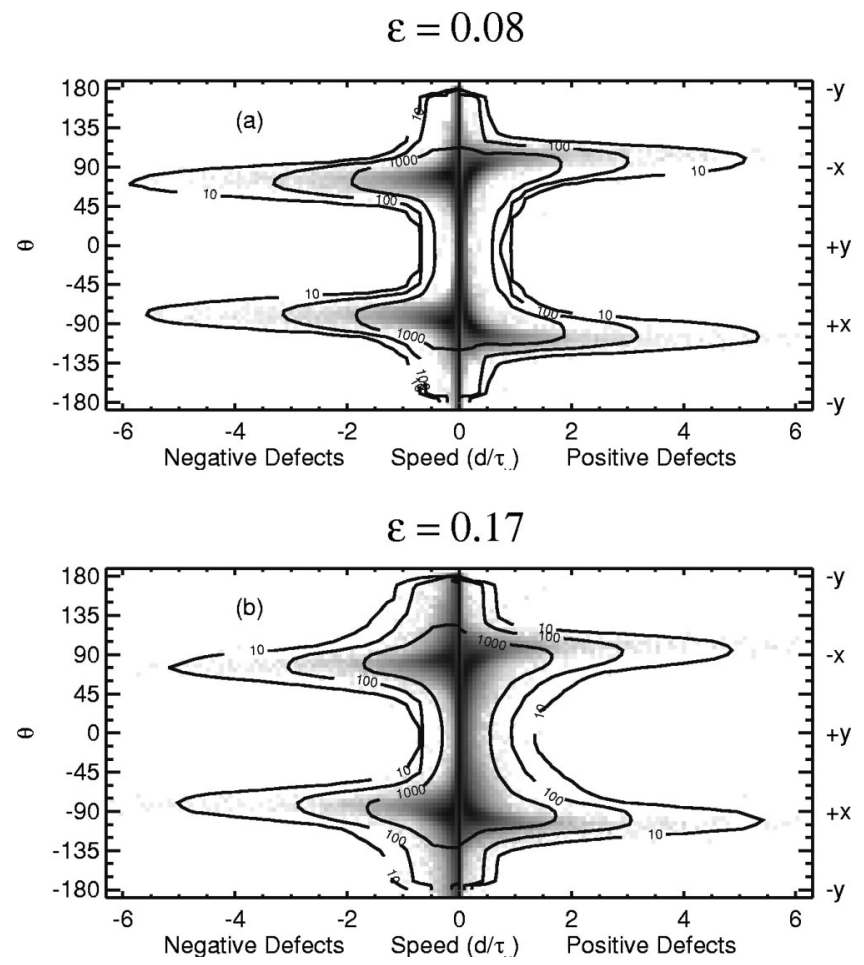

FIG. 10. Histogram for $(v, \theta)$ pairs. Negative defects plotted with negative speed and positive defects plotted with positive speed. Gray scale is shown on a logarithmic scale. (a) $\epsilon=0.08$; (b) $\epsilon=0.17$. 


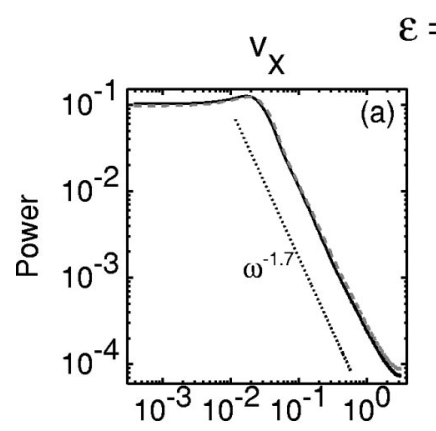

$\omega$

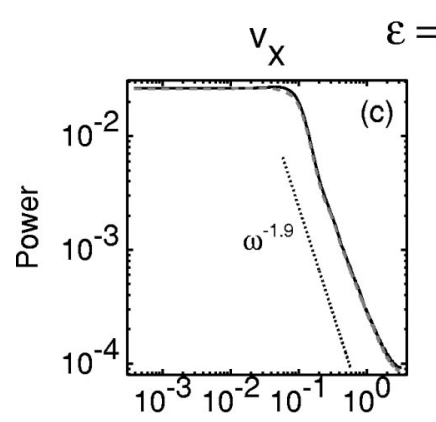

$\omega$

$$
\varepsilon=0.08
$$

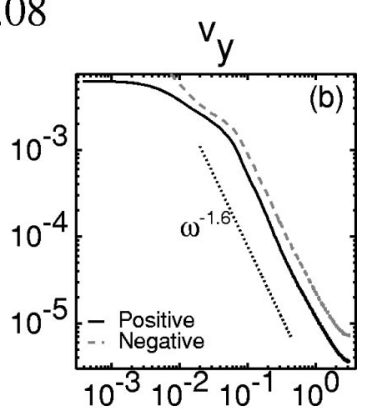

$\omega$

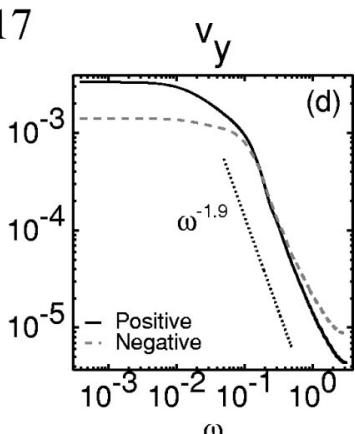

$\omega$
FIG. 11. Power spectra of $(\mathrm{a}, \mathrm{c}) v_{x}$ and $(\mathrm{b}, \mathrm{d}) v_{y}$ at $\epsilon=0.08$ and 0.17 for positive (black solid) and negative (gray dashed) defects. Dotted lines show power law fit region for positive defects.

for defects to travel other than in the preferred direction. Asymmetries in defect behavior with respect to topological charge have been observed in other anisotropic systems such as Langmuir circulations, ${ }^{27}$ although for quantities such as the surface convergence rather than the defect velocity. In this case, the asymmetry may be related to the breaking of the $\hat{\mathbf{z}}$ symmetry by non-Boussinesq effects.

In a system consisting of Lévy flight behavior, the power spectra $S(\omega)$ of $v(t)$ is expected to show the power law behavior for $\pi \gg \omega \gg \omega^{*}=1 / \Delta t^{*}$, where $\Delta t^{*}$ is a short time associated with the low- $t$ limit of power law behavior of the PDF of $\Delta t$ for flights. ${ }^{19}$ Such a cutoff is relatively large $\left(\approx 5 \tau_{v}\right)$ in the defect flight durations shown in Fig. 7 , limiting the range over which we would expect to see power laws in $S(\omega)$ for defect trajectories. Figure 11 suggests randomwalk behavior (constant, uniform spectrum) for low $\omega$, crossing over to a flight-related power law at high $\omega$. The exponent in all cases ( $\epsilon, \hat{\mathbf{x}}, \hat{\mathbf{y}}$, and topological charges) is approximately -1.8 .

\section{DEFECT INTERACTIONS}

The defects carry topological charge, and the resulting phase fields allow them to interact with each other. By examining the relative position of nearby defects, we can gain insight into their interactions. We examine strips of width $\delta y=2 d($ or $\delta x)$ and calculate the distances $\Delta x$ (or $\Delta y$ ) separating all pairs of defects within the strip (see Fig. 12 for a schematic).

Figure 13 plots PDFs of this data for all $(+,-),(+,+)$, and $(-,-)$ pairs of defects: $\mathcal{P}(\Delta x \mid \delta y<2 d)$ and $\mathcal{P}(\Delta y \mid \delta x$ $<2 d)$. The data are limited by the finite size of the region under consideration, and are only plotted over half the di-

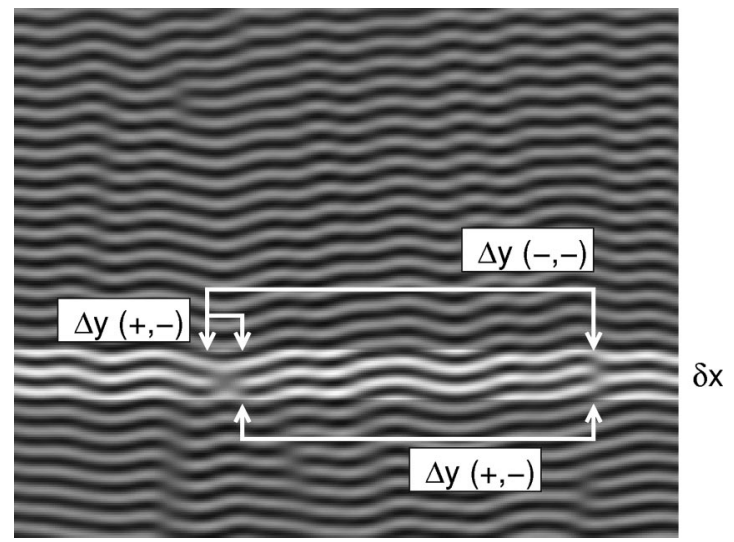

FIG. 12. Schematic diagram of determination of defect pair separations in $\hat{\mathbf{y}}$, with associated $\Delta y$ measurements.

mensions of the region. For both $\epsilon$, it is rare for oppositely charged defects to be located within $3 d$ of each other. This is associated with the annihilation of such pairs which pass too close in either direction.

Defect pairs of $(+,+)$ or $(-,-)$ charge behave in similar fashions to each other, demonstrated by the closely overlapping dashed and dotted lines of Fig. 13. There is a repulsive effect in the $\hat{\mathbf{x}}$ direction, with $\Delta x$ increasing away from 0 . The turnover in the $\epsilon=0.17$ PDF may be due to finite size considerations. In the $\hat{\mathbf{y}}$ direction, there is again an excluded region of around $5 d$, an effect which is strong enough that secondary and tertiary peaks at $10 d$ and $15 d$ are visible as well for $\epsilon=0.17$.

$$
\varepsilon=0.08
$$
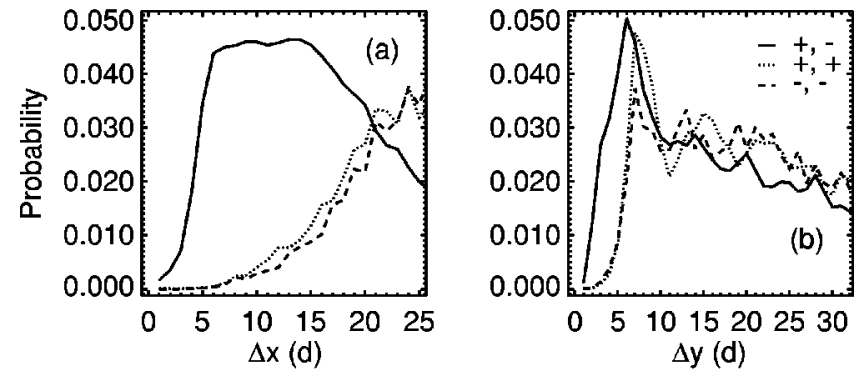

$\varepsilon=0.17$
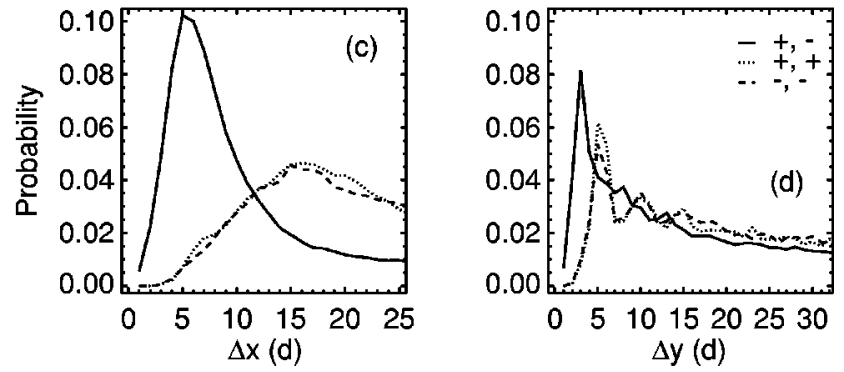

FIG. 13. PDF of defect pair separations ( $\Delta x$ and $\Delta y$ ) for strips of width $\delta y=\delta x=2 d$ as shown in Fig. 12. (a,c) Transverse separations and (b,d) longitudinal separations. Solid lines are $(+,-)$ pairs, dotted are $(+,+)$, and dashed are $(-,-)$. Approximately $10^{5}$ elements used for each curve. 


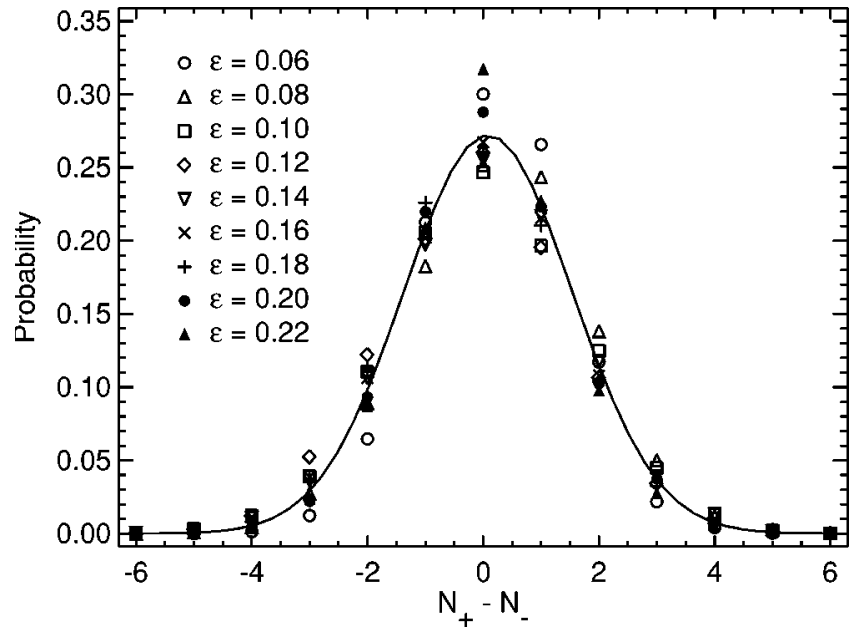

FIG. 14. PDF of net charge $\left(N_{+}-N_{-}\right)$found in the subregion for nine values of $\epsilon$. Solid line is a Gaussian fit.

\section{CREATION AND ANNIHILATION EVENTS}

A general feature of defect turbulent systems is the production and loss of defects through two mechanisms: pair creations/annihilations and flux through the boundaries. A mean-field approximation has been postulated by Gil et al. ${ }^{10}$ and experimentally examined by Falcke $e t a l^{8}$ and Daniels and Bodenschat ${ }^{15}$ in which these rates depend on the number of defects in the system:

$$
\begin{aligned}
& C(N)=C_{0}, \\
& E(N)=E_{0}, \\
& L(N)=L_{0} N, \\
& A(N)=A_{0} N^{2},
\end{aligned}
$$

where $C(N)$ is the probability of a pair creation event happening per unit time, $A(N)$ is pair annihilation, $L(N)$ is a single defect leaving, and $E(N)$ is a single defect entering. $N$ is taken to be either the number of positive defects $N_{+}$or the number of negative defects $N_{-}$, quantities which are approximately equal on average. Figure 14 shows PDFs of the net charge $\left(N_{+}-N_{-}\right)$for the observed images, with a mean of zero (topologically neutral), independent of $\epsilon$. The constant creation and entering rates can be understood as being generated by random events, independent of the number of defects already in the system. The annihilation rate scales as the number of positive defects times the number of negative defects. The leaving rate is proportional to the number of defects present in the system.

Based on this approach, we earlier derived and tested a universal distribution for $N$ which agrees with the experimental findings. ${ }^{15}$ We find, as well, that the subscripted coefficients depend on $\epsilon$, system size, and other physical parameters in systematic ways.

The stationary distribution for $N$ was found using a recursive relation,

$$
\operatorname{loss}(N) \mathcal{P}(N)=\operatorname{gain}(N-1) \mathcal{P}(N-1)
$$

to describe the probabilities at adjacent $N .{ }^{15}$ In fact, the more stringent condition of detailed balance holds as well. By
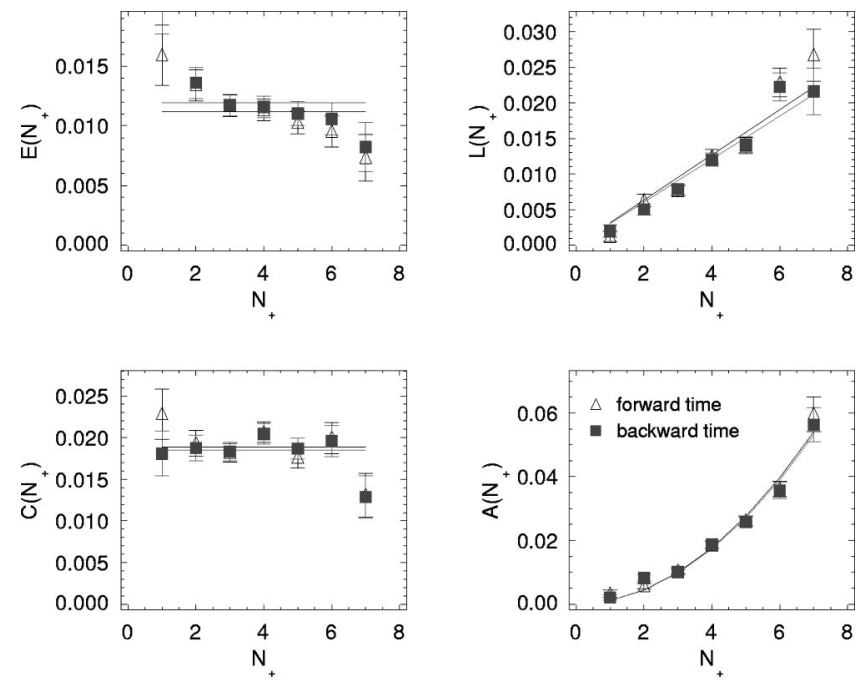

FIG. 15. Comparison of gain/loss coefficients for forward-time (triangles) and backward-time (squares) trajectories at $\epsilon=0.07$. Lines are fits to the expected $N$-dependence as described in text.

time-reversing all trajectories, creations become annihilations and entering defects leave the subregion. Analysis of the reversed trajectories for $\epsilon=0.07$ is shown in Fig. 15 in comparison with the original data and found to exhibit the same behavior.

Such defect gain and loss rates properly scale with the size of the region under consideration. We define a set of size-independent coefficients $c_{0}, a_{0}, e_{0}$, and $l_{0}$ to quantify this behavior. Both creation and entering are random events, which have some rate per unit area and length, respectively. Thus, $c_{0} \equiv C(N) / S$ and $e_{0} \equiv E(N) / P$, where $S$ and $P$ are the surface area and perimeter of the subregion, respectively. With primed and unprimed variables representing different subregion sizes, $c_{0} \equiv C_{0} / S=C_{0}^{\prime} / S^{\prime}$ and $e_{0} \equiv E_{0} / P$ $=E_{0}^{\prime} / P^{\prime}$. For two subregions with the same density of defects $n \equiv N / S$, the annihilations per unit area (at constant $n$ ) is also constant. For $A(N)=A_{0} N^{2}=A_{0} n^{2} S^{2}=A_{0}^{\prime} n^{2} S^{\prime 2}$ and constant density of defects $n, a_{0} \equiv[A(N) / S] \propto A_{0} S$. Analogously, $l_{0} \equiv L_{0} P$.

Figure 16 compares these rescaled coefficients for various sizes of test regions. Because of the $\hat{\mathbf{x}}-\hat{\mathbf{y}}$ anisotropy, regions with the same aspect ratio as the homogeneous subregion were used. All four coefficients show constant rescaled rates for a given $\epsilon$. There is, however, a slight trend in the annihilation rates; larger boxes allow more defects to find each other and annihilate, particularly at higher $\epsilon$. This may also relate to the larger test regions approaching the less homogeneous sidewall regions (see Ref. 16).

Figure 17 shows the coefficients $C_{0}, E_{0}, L_{0}$, and $A_{0}$ as a function of $\epsilon$. The error bars were determined via a bootstrap method; by resampling the data with replacement we obtained a distribution of values for each coefficient from which to estimate the error. At present we have no explanation for the particular shapes of these graphs, which appear to be linear to first order. Both $C_{0}(\epsilon)$ and $E_{0}(\epsilon)$ appear to intercept the $\epsilon$-axis close to the $\epsilon_{c} \approx 0.02$ onset of undulation chaos. ${ }^{15}$ For $A_{0}(\epsilon)$ and $L_{0}(\epsilon)$, both lines extrapolate to the origin, the expected behavior for $N=0$. The data at $\epsilon=0.04$ 

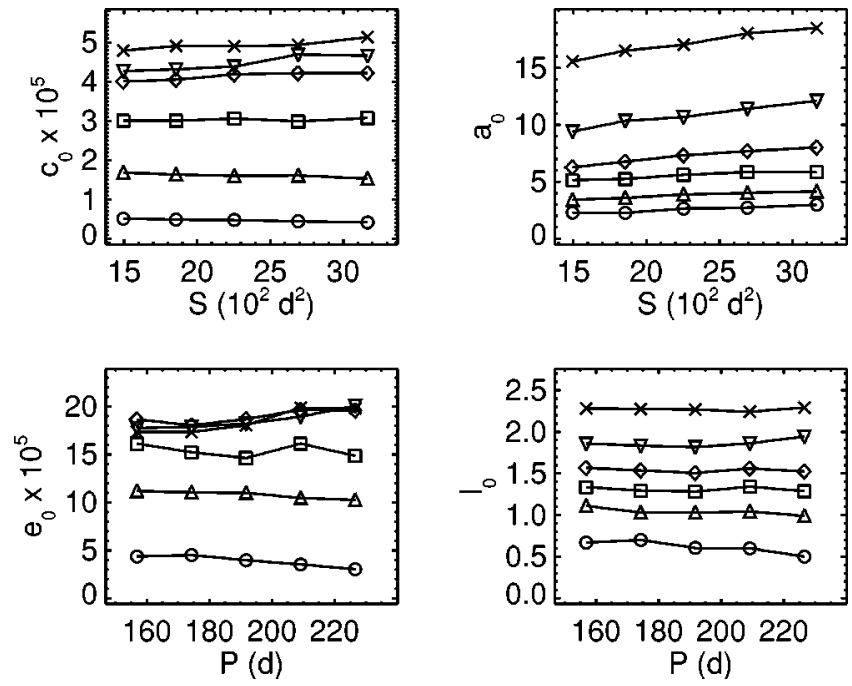

FIG. 16. Size-independent gain/loss coefficients versus size of test region. $\epsilon$ from bottom to top are $0.06(\bigcirc), 0.09(\triangle), 0.12(\square), 0.15(\diamond), 0.18(\nabla)$, $0.22(\times)$. Data are shown for positive defects; results for negative defects were similar.

and 0.05 have been disregarded due to finite-size effects which are significant at low values of $\epsilon$ since the undulations are a long-wavelength $(k=0)$ instability. Defects are increasingly rare for $\epsilon \longrightarrow \epsilon_{c}$ as the wavelength of the undulations becomes on the order of the size of the convection cell.

\section{CONCLUSION}

The defect trajectories observed in inclined layer convection display many intriguing behaviors. The occasional rapid motion of defects across many convection rolls can be associated with power laws in various quantitative measures of their motility: diffusion, velocity PDFs, flight size PDFs, and velocity power spectra. While the system is strongly anisotropic and its defect motions are dominated by transverse motion, PDFs of the defect separations reveal significant correlations in the longitudinal direction as well. Finally, the trajectories allow us to probe the gain and loss of defects in which much of this interesting behavior can be averaged over to reveal general results.

We have focused primarily on characterizing the observed flight behavior. If these rapid motions are, in fact, related to Lévy flights then the exponents found in each of the power laws should be related to each other as has been presented in the literature for various random walk formalisms. ${ }^{18,19,28}$ However, comparisons to existing theory are difficult due to both the strongly anisotropic nature and the finite size of this system. Further investigation into this phenomenon, possibly in other systems, will undoubtedly be fruitful.

The trajectories analyzed here were obtained for $\epsilon=0.08$ and 0.17 , of which the former is in a state of undulation chaos and the later intermittently exhibit ordered undulations. The dynamics of this transition is discussed in Ref. 16, and the defect trajectories should also be examined in light of the existence of the two states. The statistics presented here will presumably vary according to whether the system is
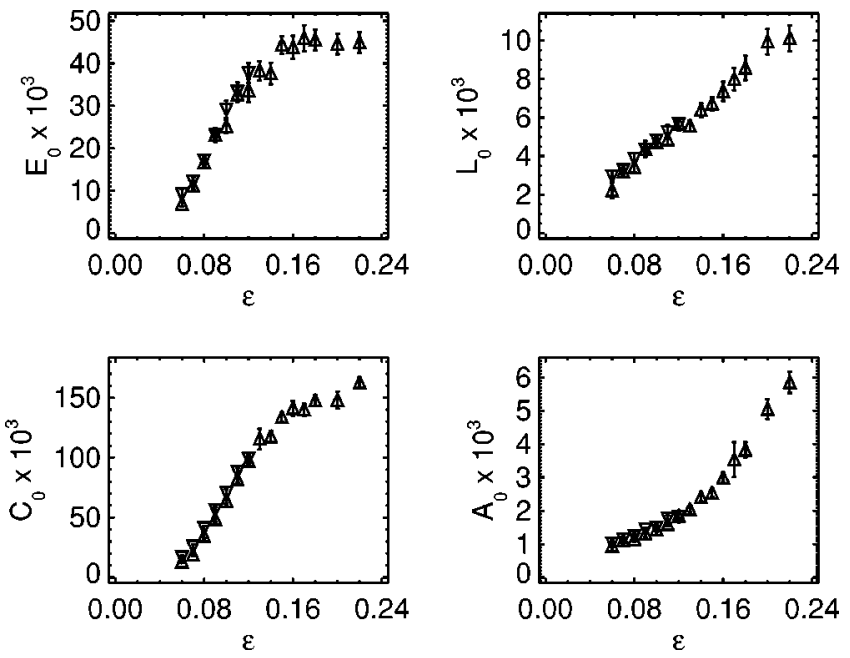

FIG. 17. Rate coefficients as a function of $\epsilon$ for the homogeneous subregion. $\triangle$ represent data taken under quasistatic temperature increase; $\nabla$ represent data taken under quasistatic temperature decrease.

in an ordered or disordered state. Furthermore, the motion of the defects during the transition may shed light on the nature of the transition.

Other questions remain regarding the relation between the defect motion and the underlying undulation pattern. For instance, the relationship between the motion of the defects and the stability of the local wavenumber. A related issue is that defects have been observed to "bounce" off regions of ordered undulations, sharply reversing direction.

Finally, the relationship between the relative position and relative velocity of defects has not yet been investigated. Further work in this area would provide information about the attraction and repulsion of defects, particularly near creation and annihilation events.

\section{ACKNOWLEDGMENTS}

We are grateful to G. M. Zaslavksy, T. Geisel, J. P. Sethna, C. L. Henley, C. Beck, and N. Mordant for useful discussions and the NSF for support under No. DMR0072077.

${ }^{1}$ M. C. Cross and P. C. Hohenberg, Rev. Mod. Phys. 65, 851 (1993).

${ }^{2}$ P. Coullet, L. Gil, and J. Lega, Phys. Rev. Lett. 62, 1619 (1989).

${ }^{3}$ I. Rehberg, S. Rasenat, and V. Steinberg, Phys. Rev. Lett. 62, 756 (1989).

${ }^{4}$ P. Ramazza, S. Residori, G. Giacomelli, and F. Arecchi, Europhys. Lett. 19, 475 (1992).

${ }^{5}$ A. La Porta and C. M. Surko, Physica D 139, 177 (2000).

${ }^{6}$ S. W. Morris, E. Bodenschatz, D. S. Cannell, and G. Ahlers, Phys. Rev. Lett. 71, 2026 (1993).

${ }^{7}$ Q. Ouyang and J. M. Flesselles, Nature (London) 379, 143 (1996).

${ }^{8}$ M. Falcke, M. Bar, J. D. Lechleiter, and J. L. Hudson, Physica D 129, 236 (1999).

${ }^{9}$ T. M. Haeusser and S. Leibovich, Phys. Rev. Lett. 79, 329 (1997).

${ }^{10}$ L. Gil, J. Lega, and J. L. Meunier, Phys. Rev. A 41, 1138 (1990).

${ }^{11}$ G. D. Granzow and H. Riecke, Phys. Rev. Lett. 87, 174502 (2001).

${ }^{12}$ B. Echebarria and H. Riecke, Phys. Rev. Lett. 84, 4838 (2000).

${ }^{13}$ E. Bodenschatz, W. Pesch, and G. Ahlers, Annu. Rev. Fluid Mech. 32, 709 (2000)

${ }^{14}$ K. E. Daniels, B. B. Plapp, and E. Bodenschatz, Phys. Rev. Lett. 84, 5320 (2000)

${ }^{15}$ K. E. Daniels and E. Bodenschatz, Phys. Rev. Lett. 88, 034501 (2002).

${ }^{16}$ K. E. Daniels, O. Brausch, W. Pesch, and E. Bodenschatz (in preparation). 
${ }^{17}$ J. Klafter, M. F. Shlesinger, and G. Zumofen, Phys. Today 49 (2), 33 (1996).

${ }^{18}$ M. F. Shlesinger, G. M. Zaslavsky, and J. Klafter, Nature (London) 363, 31 (1993).

${ }^{19}$ T. Geisel, in Lévy Flights and Related Topics in Physics, Lecture Notes in Physics, edited by M. F. Shlesinger, G. M. Zaslavsky, and U. Frisch (Springer, Berlin, 1995), pp. 153-173.

${ }^{20}$ R. M. Clever and F. H. Busse, J. Fluid Mech. 81, 107 (1977).

${ }^{21}$ J. R. de Bruyn et al., Rev. Sci. Instrum. 67, 2043 (1996).
${ }^{22}$ S. P. Trainoff and D. S. Cannell, Phys. Fluids 14, 1340 (2002).

${ }^{23}$ J. P. Bouchaud and A. Georges, Phys. Rep. 195, 127 (1990).

${ }^{24}$ G. M. Zaslavsky et al., Phys. Plasmas 7, 3691 (2000).

${ }^{25}$ T. H. Solomon, E. R. Weeks, and H. L. Swinney, Phys. Rev. Lett. 71, 3975 (1993).

${ }^{26}$ C. Beck, Phys. Rev. Lett. 87, 180601 (2001); (private communication).

${ }^{27}$ R. Bhaskaran and S. Leibovich, Phys. Fluids 14, 2557 (2002).

${ }^{28}$ E. R. Weeks and H. L. Swinney, Phys. Rev. E 57, 4915 (1998). 